RESEARCH

\title{
RELATIONSHIPS BETWEEN PARKINSON'S DISEASE DEMENTIA AND 25-HYDROXY VITAMIN D AND BRAIN-DERIVED NEUROTROPHIC FACTOR LEVELS
}

Turkish Journal of Geriatrics

DOI: 10.31086/tigeri.2021.238

2021; 24(3): 407-415

- Tuba EKMEKYAPAR ${ }^{1}$

- Yusuf TÜRKÖZ2

- Zeynep ERDEML ${ }^{3}{ }^{3}$

- Yasemin DEMIRTAŞ ${ }^{4}$

- Sibel ALTINAYAR ${ }^{4}$.

\section{CORRESPONDANCE}

\section{${ }^{1}$ Tuba EKMEKYAPAR}

Malatya Training and Research Hospital,

Department of Neurology, Malatya, Turkey

Phone: +905321626986

e-mail: dr.tuba_polat@hotmail.com

Received: Apr 15, 2021

Accepted: Aug 24, 2021

${ }^{1}$ Malatya Training and Research Hospital, Department of Neurology, Malatya, Turkey

2 Inonu University, School of Medicine, Department of Biochemistry, Malatya, Turkey

${ }^{3}$ Malatya Training and Research Hospital, Department of Biochemistry, Malatya, Turkey

${ }^{4}$ Inonu University, School of Medicine,

Department of Neurology, Malatya, Turkey

\section{Abstract}

Introduction: Parkinson's disease dementia is an important condition that worsens the quality of life in approximately $30 \%$ of parkinson disease patients. Vitamin $\mathrm{D}$ deficiency and brain-derived neurotrophic factor have been implicated in the pathogenesis of alzheimer type dementia, parkinson's disease, and many neurodegenerative diseases. The aim of this study was to investigate the relationship between cognitive impairment and 25-hydroxy vitamin $\mathrm{D}$ and brain-derived neurotrophic factor levels in parkinson's disease dementia patients.

Materials and Method: 25-hydroxy vitamin D and brain-derived neurotrophic factor serum levels were evaluated in patients with parkinson's disease dementia $(n=63)$ and healthy controls $(n=33)$. Brain-derived neurotrophic factor and vitamin $D$ levels were examined using one sample t-tests, and multiple comparisons among independent groups were done using ANOVA post hoc Tukey's test analysis. Wechsler Memory Scale and MiniMental State Examination were used to evaluate the cognitive functions of the groups. The disease levels of the patients were determined using the Hoehn and Yahr scale and the Unified Parkinson's Disease Rating Scale.

Results: There was a significant negative correlation between the worsening of dementia and vitamin $D$ levels $(p=0.009)$. However, there were significant negative correlations between the unified parkinson's disease rating scale daily living activity and Hoehn and Yahr scales and vitamin D levels. No significant relationship was found between brain-derived neurotrophic factor and parkinson's disease dementia ( $p=0.983)$.

Conclusions: Vitamin D deficiency plays a role in cognitive loss in parkinson's disease dementia. Vitamin D replacement can be used in dementia support treatment.

Keywords: Parkinson Disease; Dementia; Vitamin D; Brain-Derived Neurotrophic Factor. 


\section{INTRODUCTION}

Idiopathic Parkinson's disease (IPD) is a progressive neurodegenerative disease that is accompanied by resting tremors, bradykinesia, rigidity, and postural disorders (1). Non-motor signs are also common in IPD but are mostly ignored. The incidence of IPD in all age groups ranges from 4.5 to 9 per 100,000 (2). Cognitive loss and dementia develop over the course of IPD in $30 \%$ of patients $(3,4)$. Parkinson's disease dementia (PDD) constitutes $0.5 \%$ of all dementia cases in people over the age of 65 and 3-4\% of the dementia cases in all age groups $(5,6)$. Symptoms like rigidity and akinesia, recurring falls, and severe gait disorders are significant risk factors for slow-onset, severe cognitive deterioration (7). Frontal executive function disorders and memory errors are the main characteristics of the disease (7).

Vitamin $D$ is a neuro-steroid hormone that has important roles in bone mineralization and the regulation of the calcium-phosphorus balance (8). Vitamin D has significant effects on the brain and has various roles in the central nervous system, in cell proliferation and differentiation, neurotransmission, and neuroplasticity as well as neuroprotection (9).

Brain-derived neurotrophic factor (BDNF) is a member of the neurotrophin family and is found in several brain regions (10). BDNF mainly affects neuron development and regeneration and the structural health of major neural pathways and their functioning. A correlation has been found between low BDNF levels and alzheimer's disease $(11,12)$. It is believed that BDNF is also associated with other neurodegenerative diseases, including IPD (13).

The aim of this study was to investigate the relationship between cognitive impairment and 25-hydroxy vitamin $\mathrm{D}(25(\mathrm{OH})$ vitamin $\mathrm{D})$ and $\mathrm{BDNF}$ levels in PDD patients.

\section{MATERIALS AND METHODS}

\section{Patient Selection}

The study included 63 patients with IPD who were followed up in the Movement Disorders Clinic of the Department of Neurology, Inonu University, and 33 healthy individuals of similar age and education level without any neurological disease. There were no patients diagnosed with osteoporosis in either group. Individuals were diagnosed using their files and clinical evaluations. None of the participants were using vitamin $D$, multivitamins, or calcium or phosphate group drugs.

The specimens for measuring the BDNF levels of 13 patients had deteriorated, and these patients were excluded. Subjects who were on any vitamin replacement treatment, those with a bone metabolism disease, such as osteoporosis, or a chronic systemic disease, were excluded. All subjects provided informed consent before participating in the study, which was approved with a protocol code of 2016/23 by the Malatya Clinical Research Ethics Committee.

The personal and actual knowledge and longterm memory of all subjects were assessed using the Wechsler Memory Scale (WMS) 1; orientation was assessed with WMS 2; mental control and the ability to sustain attention were assessed with WMS 3; logical memory (momentary and long-term remembering test) was assessed with WMS 4; and WMS R was used to assess simple and complex attention based on numbers and visual range. Proverb interpretation and similarity tests were used to assess abstract thinking (abstraction); planning skills were assessed by drawing clocks; structuring was assessed by shape copying; concentric circles were drawn to evaluate constructional praxis; and verbal conciseness functions were examined via word finding. For the patients who could not perform these tests due to cognitive loss, the Mini-Mental State 
Examination (MMSE) was administered. In this test, participants answer 11 questions and can obtain a maximum of 30 points; scores of $20-24$ points indicate mild cognitive disorder, 10-19 indicate moderate dementia, and less than 10 points, severe dementia. For the patients whose clinical dementia scores were calculated, orientation, memory, attention, calculation, recall, language, motor function and perception, and visuospatial capacities were evaluated.

After undergoing the neurological examinations, the patients' disease levels were determined using the Hoehn and Yahr (H\&Y) scale and the Unified Parkinson's Disease Rating Scale (UPDRS).

\section{Blood Samples}

Fasting blood samples were collected in vacutainers and centrifuged at $3000 \mathrm{rpm}$ for $10 \mathrm{~min}$ to obtain serum. The serum samples were frozen at $-70^{\circ} \mathrm{C}$ until the BDNF and $25(\mathrm{OH})$ Vitamin D levels were measured. Before the analysis, the frozen serum samples were slowly brought to room temperature and stirred gently. The BDNF and 25(OH) vitamin $D$ analyses were performed on the same day.

\section{Biochemical Analysis of Serum BDNF}

The serum BDNF levels of both groups were measured using a commercial enzyme-linked immunosorbent assay (ELISA) kit (Boster Biological Technology, Pleasanton, CA, USA) based on the manufacturer's instructions. This analysis is based on the ELISA method. The serum and standard samples were added to wells that were precoated with human BDNF monoclonal antibodies and incubated for $90 \mathrm{~min}$ at $37^{\circ} \mathrm{C}$. After incubation, the contents of the wells were removed by overturning the plate on a paper towel. After this, biotinylated polyclonal detection antibodies were added to each well and incubated for $60 \mathrm{~min}$ at $37^{\circ} \mathrm{C}$. After incubation, the wells were washed three times to extract unbound biotinylated polyclonal antibodies. After this, an avi- din-biotin-peroxidase complex was added to each well and incubated for $30 \mathrm{~min}$ at $37^{\circ} \mathrm{C}$. After incubation, the wells were washed three times to extract the unbound avidin-biotin-peroxidase complex. A tetramethylbenzidine (TMB) solution (chromogen) was then added to each well and incubated in the dark for $25-30 \mathrm{~min}$ at $37^{\circ} \mathrm{C}$, and thus, the color of the fluid in the wells turned blue. The reaction was ended by adding a TMB stop solution (acidic stop solution), and the blue color finally turned yellow. The absorbance values of the yellow fluid were measured at $450 \mathrm{~nm}$ using a microplate reader (Synergy H1 Hybrid Multi-Mode Reader; BioTek Instruments, Inc., Winooski, VT). The absorbance of the yellow color that was obtained was proportional to the level of human BDNF found in the serum. The results are reported in units of $\mathrm{pg} / \mathrm{mL}$. The intra-assay and inter-assay coefficient of variation values of the test were $<4.5 \%$ and $<7.5 \%$, respectively.

\section{Biochemical Analysis of Serum Vitamin D}

The serum vitamin D levels were analyzed using high-performance liquid chromatography (HPLC) with a Shimadzu LC-10ADVP HPLC system (Shimadzu Corporation, Kyoto, Japan), Immuchrom vitamin D3 controls, and an HPLC kit (Immuchrom GmbH, Heppenheim, Germany) while measuring the serum $25(\mathrm{OH})$ vitamin D levels. In summary, this method is based on subjecting samples to precipitation and extraction before they are injected into the HPLC system. The HPLC separation process was carried out with a 'reverse phase' C18, $150 \times 4.6 \mathrm{~mm}$ particle-size column (VertiSep GES; Vertical Chromatography Co., Ltd., Thailand) at $30^{\circ} \mathrm{C}$ at a $1 \mathrm{~mL} / \mathrm{min}$ flow rate and using acetonitrile-water $(99: 1, \mathrm{v} / \mathrm{v})$ as the mobile phase. The volume of the injected samples was $50 \mu \mathrm{l}$, and the HPLC separation process lasted for $15 \mathrm{~min}$. Chromatograms were determined using a UV detector at $264 \mathrm{~nm}$. The results were calculated with the 'in-house standard method' via the integration of the peak regions. The results are reported as $\mathrm{nmol} / \mathrm{L}$. The coefficient of variation values 
of the test were $<2.5 \%$ and $<4.0 \%$ for intra-assay and inter-assay, respectively.

\section{Statistical Analysis}

Statistical analysis was performed using SPSS v.17 software (SPSS Inc., Chicago, IL, USA). The data were analyzed using SPSS, and $p<0.05$ was considered significant. The differences between the groups in terms of age, education level, disease duration, age of disease onset, and $25(\mathrm{OH})$ vitamin $\mathrm{D}$ and BDNF levels were examined using a t-test. The differences in the gender ratios between the groups were calculated using the chi-square test. Analysis of variance (ANOVA) and the post hoc Tukey's test were used for multiple comparisons among independent groups, while the cognitive levels and H\&Y stages of the groups were compared using the Pearson's chi-square test. The Pearson's correlation test was used to determine the correlation between the UPDRS score and $25(\mathrm{OH})$ vitamin D and BDNF levels.

\section{RESULTS}

The mean age of the patients was $65.54 \pm 7.07$ years versus $66.88 \pm 5.37$ years for the controls $(p=0.097$ )
There was no significant gender difference between the groups ( $p=0.101)$. The normal range of the $25(\mathrm{OH})$ vitamin $\mathrm{D}$ levels for both groups was accepted as $75-250 \mathrm{nmol} / \mathrm{L}$. The mean $25(\mathrm{OH})$ vitamin $\mathrm{D}$ level was $240.55 \pm 89.01 \mathrm{nmol} / \mathrm{L}$ for the patients and $217.03 \pm 68.71 \mathrm{nmol} / \mathrm{L}$ for the controls $(p=0.146)$. Similarly, there was no significant difference in the BDNF levels of the two groups ( $p=0.604$ ) (Table 1).

For the patients, the mean disease duration was $5.32 \pm 3.75$ years, and the mean age of disease onset was $60.13 \pm 7.12$ years. Comparing the cognitive levels of the patients and UPDRS scores, the UPDRS-cognitive function score was significantly correlated with the stage of dementia $(p<0.001)$ (Table 2).

In PDD patients, there was a strong correlation between the worsening of cognitive function and a reduction in $25(\mathrm{OH})$ vitamin $D$ levels $(p=0.009$, Table 3). However, no significant relationship was found between the BDNF levels and cognitive levels of the patients ( $p=0.983$, Table 3 ).

There was also an inverse correlation between worsening $\mathrm{H} \& \mathrm{Y}$ stage and $25(\mathrm{OH})$ vitamin D levels ( $p=0.046$, Table 4). There was no significant relationship between BDNF level and H\&Y stage $(p=$

Table 1. Comparison of age, gender, $25(\mathrm{OH})$ vitamin D, and BDNF levels between patient and control groups

\begin{tabular}{|c|c|c|c|c|}
\hline & Patient group $(n=63)$ & Control group $(n=33)$ & $\mathbf{F}$ & $p$ \\
\hline Age (years) & $65.54 \pm 7.07$ & $66.88 \pm 5.37$ & 2.808 & $0.097^{*}$ \\
\hline Vitamin D (nmol/L) & $240.55 \pm 89.01$ & $217.03 \pm 68.71$ & 2.147 & $0.146^{*}$ \\
\hline BDNF (pg/mL) & $137.80 \pm 12.08$ & $137.22 \pm 16.67$ & 0.271 & $0.604^{*}$ \\
\hline \multicolumn{5}{|l|}{ Gender } \\
\hline Female & $29(\% 58.0)$ & $21(\% 42.0)$ & 2.689 & $0.101^{\uparrow}$ \\
\hline Male & $34(\% 73.9)$ & $12(\% 26.1)$ & & \\
\hline
\end{tabular}

*t-test, "chi-square test 
Table 2. Comparison of the patients' UPDRS scores and cognitive levels

\begin{tabular}{|c|c|c|c|c|}
\hline & $\begin{array}{c}\mathrm{MCl} \\
(\mathrm{n}=36) \\
\mathrm{X} \pm \mathrm{SD}\end{array}$ & $\begin{array}{l}\text { Mild Dementia } \\
\begin{array}{l}(n=19) \\
X \pm S D\end{array}\end{array}$ & 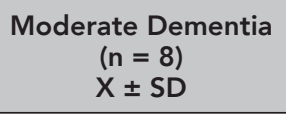 & Test and Significance \\
\hline UPDRS-Motor & $22.47 \pm 13.23$ & $25.94 \pm 12.43$ & $32.62 \pm 12.92$ & $\begin{array}{l}F=2.112 \\
p=0.130\end{array}$ \\
\hline UPDRS-Daily Life Action & $8.41 \pm 5.88$ & $9.68 \pm 5.18$ & $13.62 \pm 6.78$ & $\begin{array}{l}F=2.662 \\
p=0.078\end{array}$ \\
\hline UPDRS-Cognitive Function & $3.16 \pm 1.63$ & $4.21 \pm 1.03$ & $6.25 \pm 1.66$ & $\begin{array}{l}F=14.885 \\
p<0.001\end{array}$ \\
\hline UPDRS-Treatment Complication & $8.75 \pm 5.26$ & $8.15 \pm 4.98$ & $11.37 \pm 6.04$ & $\begin{array}{l}F=1.082 \\
p=0.345\end{array}$ \\
\hline UPDRS-Total & $42.80 \pm 23.47$ & $48.00 \pm 21.7$ & $63.87 \pm 24.13$ & $\begin{array}{l}F=2.756 \\
p=0.072\end{array}$ \\
\hline
\end{tabular}

Table 3. Comparison of cognitive functions and $25-\mathrm{OH}$ vitamin $\mathrm{D}$ and BDNF levels in patient group

\begin{tabular}{|c|c|c|c|}
\hline & $25-\mathrm{OH}$ vitamin $\mathrm{D}(\mathrm{nmol} / \mathrm{L})$ & $\mathrm{F}$ & $p$ \\
\hline $\mathrm{MCl}(n=36)$ & $269.63 \pm 89.50$ & \multirow{4}{*}{5.166} & \multirow{4}{*}{0.009} \\
\hline Mild Dementia $(n=19)$ & $205.94 \pm 76.39$ & & \\
\hline Moderate Dementia $(n=8)$ & $191.87 \pm 69.33$ & & \\
\hline \multirow[t]{2}{*}{ Total $(n=63)$} & $240.55 \pm 89.01$ & & \\
\hline & BDNF $(p g / m L)$ & $\mathrm{F}$ & $p$ \\
\hline $\mathrm{MCl}(n=29)$ & $139.90 \pm 113.06$ & \multirow{4}{*}{0.017} & \multirow{4}{*}{$0.983 Y$} \\
\hline Mild Dementia( $(\mathrm{n}=16)$ & $133.10 \pm 148.63$ & & \\
\hline Moderate Dementia(n = 5) & $140.64 \pm 80.76$ & & \\
\hline Total $(n=50)$ & $137.80 \pm 12.08$ & & \\
\hline
\end{tabular}

YANOVA Post-Hoc Tukey test 
Table 4. Comparison of H\&Y stages and $25-\mathrm{OH}$ vitamin $\mathrm{D}$ levels in patient group

\begin{tabular}{|c|c|c|c|}
\hline & $25-\mathrm{OH}$ vitamin D (nmol/L) & $\mathbf{F}$ & $p$ \\
\hline H\&Y Stage $1(n=18)$ & $267.05 \pm 88.35$ & \multirow{4}{*}{3.247} & \multirow{4}{*}{0.046} \\
\hline H\&Y Stage $2(n=31)$ & $247.38 \pm 88.22$ & & \\
\hline H\&Y Stage $3(n=14)$ & $191.35 \pm 76.97$ & & \\
\hline Total $(n=63)$ & $240.55 \pm 89.01$ & & \\
\hline
\end{tabular}

"ANOVA Post-Hoc Tukey test

Table 5. Correlation analysis of patients' UPDRS scores with vitamin D and BDNF levels

\begin{tabular}{|c|c|c|c|c|c|c|c|}
\hline & 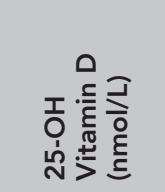 & 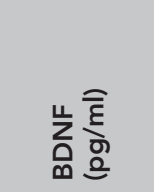 & 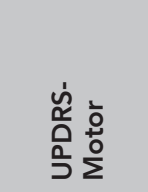 & 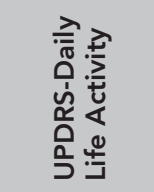 & 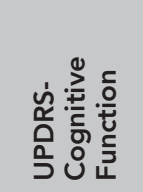 & 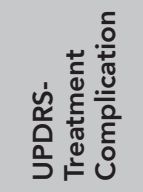 & 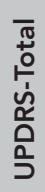 \\
\hline 25-OH Vitamin D (nmol/L) & 1 & & & & & & \\
\hline BDNF (pg/ml) & $\begin{array}{c}0,016^{\star \star} \\
(p=0,912)\end{array}$ & 1 & & & & & \\
\hline UPDRS-Motor & $\begin{array}{c}-0,164^{\star \star} \\
(p=0,200)\end{array}$ & $\begin{array}{c}-0,117^{\star \star} \\
(p=0,417)\end{array}$ & 1 & & & & \\
\hline UPDRS-Daily Life Activity & $\begin{array}{c}-0,310^{\star \star} \\
(p=0,014)\end{array}$ & $\begin{array}{c}-0,161^{\star \star} \\
(p=0,265)\end{array}$ & $\begin{array}{c}0,823^{\star *} \\
(p<0,001)\end{array}$ & 1 & & & \\
\hline UPDRS-Cognitive Function & $\begin{array}{c}-0,156^{\star \star} \\
(p=0,222)\end{array}$ & $\begin{array}{c}-0,051^{\star \star} \\
(p=0,723)\end{array}$ & $\begin{array}{c}0,602^{\star \star} \\
(p<0,001)\end{array}$ & $\begin{array}{c}0,624^{\star *} \\
(p<0,001)\end{array}$ & 1 & & \\
\hline UPDRS-Treatment Complication & $\begin{array}{c}-0,157^{\star \star} \\
(p=0,219)\end{array}$ & $\begin{array}{c}-0,081^{\star \star} \\
(p=0,575)\end{array}$ & $\begin{array}{c}0,721^{\star \star} \\
(p<0,001)\end{array}$ & $\begin{array}{c}0,658^{\star \star} \\
(p<0,001)\end{array}$ & $\begin{array}{c}0,451^{\star \star} \\
(p<0,001)\end{array}$ & 1 & \\
\hline UPDRS-Total & $\begin{array}{c}-0,216^{\star *} \\
(p=0,090)\end{array}$ & $\begin{array}{c}-0,128^{\star *} \\
(p=0,375)\end{array}$ & $\begin{array}{c}0,969 * * \\
(p<0,001)\end{array}$ & $\begin{array}{c}0,903^{\star \star} \\
(p<0,001)\end{array}$ & $\begin{array}{c}0,667^{\star *} \\
(p<0,001)\end{array}$ & $\begin{array}{c}0,823^{\star \star} \\
(p<0,001)\end{array}$ & 1 \\
\hline
\end{tabular}

** Pearson correlation test

0.375). When the UPDRS subscale scores of the patients and their $25(\mathrm{OH})$ vitamin D and BDNF levels were compared, an inverse correlation was found between their daily life activities and vitamin $D$ levels ( $p=0.014$, Table 5$)$, while there was no significant relationship between the vitamin D and BDNF levels and the other subscales. Accordingly, the relationship between low vitamin D levels and H\&Y stage was stronger than low vitamin $D$ levels relationship with the UPDRS subscales, except for the daily life activities subscale. 


\section{DISCUSSION}

Vitamin $D$ is a secosteroid that plays a role in the regulation of bone health physiology and calcium-phosphorus homeostasis (14). Vitamin D levels were determined by measuring serum levels of $25(\mathrm{OH})$ vitamin D. In our study, we tried to obtain reliable results by examining the $25(\mathrm{OH})$ vitamin $D$ levels, which has a half-life that can be extended when blood samples are taken under protection from light. Statistically, there was no significant difference between the $25(\mathrm{OH})$ vitamin D levels of the patients and those of the controls in this study. Both groups had mean $25(\mathrm{OH})$ vitamin $D$ levels in the normal reference range. The lack of a difference between our two groups might be explained by the fact that most of our patients lived in rural areas, were sufficiently exposed to sunlight, and consumed mostly organic foods, unlike individuals in developed countries or urban areas.

Vitamin D is a neurosteroid, and its deficiency has been the subject of research in the pathogenesis of many neurological diseases. There are many studies that examine the relationship between cognitive loss and vitamin D deficiency, especially in alzheimer's disease. In a study of 412 Korean patients, Ahn et al. found a positive correlation between vitamin D levels and MMSE scores (15). They also showed that physical activity affected vitamin $D$ levels, and independence in physical activity might indirectly affect MMSE scores (15). Llewellyn et al. examined cognitive function in elderly individuals and reported a 0.3-point loss per year in the MMSE scores of those with vitamin D deficiencies (16). Peterson et al. obtained similar results, finding positive correlations between vitamin $D$ levels and verbal conciseness and verbal memory (17). These studies have suggested that vitamin $D$ has a positive effect on cognitive function. There are few studies that examine the relationship between PDD and vitamin D. In this study, our patients were divided into three groups according to their MMSE scores: mild cognitive impairment, mild dementia, and moderate dementia. A positive correlation was observed between the MMSE scores of PDD patients and vitamin D deficiency. Although vitamin D levels were still in the normal reference range, a decrease in these levels might have contributed to the development of cognitive loss or dementia in PDD.

The relationship between the motor symptoms of parkinson's disease and vitamin D has recently been a subject of research. It has been thought that there may be many factors affecting vitamin $D$ deficiency in parkinson's disease. Knekt et al. found a significant relationship between parkinson's disease and vitamin D levels, which was affected by age, sex, marital status, education level, alcohol consumption, physical activity, smoking, and body mass index (18). In another study, Evatt et al. examined the vitamin D levels of parkinson's patients and healthy individuals and found that the vitamin $D$ levels of parkinson's patients were significantly lower (19).

Suzuki et al. also found significant correlations between the H\&Y and UPDRS total and motor scores of individuals with a diagnosis of parkinson's and the vitamin D receptor Fok1 genotype and low vitamin D levels (20). Similarly, in our study, there was a relationship between the H\&Y and UPDRS-daily life activity scores of the patients and low vitamin $D$ levels. We found a significant negative correlation between PDD and 25(OH) vitamin D levels and a negative correlation between the patients' $H \& Y$ stages and their $25(\mathrm{OH})$ vitamin D levels. However, the only relationship between the UPDRS scores and $25(\mathrm{OH})$ vitamin $D$ levels was for the daily life activities subscale.

In a meta-analysis, Zhou et al. stated that low vitamin D levels may contribute to the development of parkinson's disease, but vitamin replacement does not have a significant effect on motor function (21). However, Llewellyn et al. showed that early stage vitamin D replacement therapy is important in improving MMSE scores in elderly individuals (16). Accordingly, we think that although the measured $25(\mathrm{OH})$ vitamin $D$ levels were in the normal range, 
supplementary treatment should be started when these scales worsen, which might slow the progression of dementia rather than motor symptoms.

BDNF is a neurotrophin that contributes to the development and regeneration of neurons in the central and peripheral nervous systems (10). Howells et al. determined that BDNF expression was decreased in alzheimer's, parkinson's, and huntington's disease, amyotrophic lateral sclerosis, and multiple sclerosis (13). Also, Laske et al. compared the serum and cerebrospinal fluid BDNF levels of patients with alzheimer's disease, normal-pressure hydrocephaly, and healthy individuals and found that the BDNF levels were significantly lower in both disease groups compared to the healthy individuals (22). In another study, researchers examined 47 parkinson's patients and 23 healthy individuals and found that the serum BDNF levels were significantly lower in the patients (23). By contrast, Faria et al. examined 50 Alzheimer's patients, 37 mild cognitive disorder patients, and 56 healthy individuals and found that the plasma BDNF levels were significantly higher in the alzheimer's group (24). Hence, different results have been found regarding BDNF levels in various neurodegenerative diseases. In our study, there was no significant relationship between BDNF and PDD or disease stages. According to our current knowledge, BDNF is not suitable for predicting the course of neurodegenerative diseases. However, this needs to be clarified with larger, more comprehensive studies. When evaluating the differences in the results of the different studies, we should note that neurotrophin synthesis is influenced by genetic, environmental, and infectious factors.

This study has some limitations. We had a low number of patients and controls. Our results should be supported by studies with a larger number of patients. The absence of any severe dementia in our study may affect the results.

In conclusion, a negative correlation was found between the H\&Y stages, UPDRS-daily living activities and PDD dementia levels and vitamin D levels. These results suggest that vitamin D has an important role in cognitive functions in PDD. However, no significant difference was found in the BDNF levels between the PDD and healthy groups or based on the dementia stages of the PDD group. In this study, the idea that vitamin $D$ affects dementia pathogenesis and cognitive degeneration is supported, and early vitamin $D$ replacement can contribute to dementia prevention. More comprehensive studies are needed on this subject.

\section{Acknowledgements}

We would like to thank the Inonu University Scientific Project Unit.

\section{Financial support}

This study was supported by the Inonu University Scientific Project Unit with project number 2016-75.

\section{Conflicts of interest}

There are no conflicts of interest.

\section{Ethical approval}

This study, with a protocol code of 2016/23, was approved by the Malatya Clinical Research Ethics Committee.

\section{Informed consent}

Written informed consent was obtained from all individual participants included in the study. 


\section{REFERENCES}

1. Massano J, Bhatia KP. Clinical Approach to Parkinson's Disease: Features, Diagnosis, and Principles of Management. Cold Spring Harb Perspect Med.2012; 2 (6): a008870. (PMID: 22675666).

2. Van Den Eeden SK, Tanner CM, Bernstein AL, et al. Incidence of Parkinson's disease: variation by age, gender, and race/ethnicity. Am J Epidemiol 2003; 157 (11): 1015-22(PMID: 12777365).

3. Aarsland D, Zaccai J, Brayne C. A systematic review of prevalence studies of dementia inParkinson's disease. Mov Disord 2005; 20 (10): 1255-63. (PMID: 16041803).

4. Riedel O, Klotsche J, Spottke A, et al. Frequency of dementia, depression, and other neuropsychiatric symptoms in 1,449 outpatients with Parkinson's disease. J Neurol 2010; 257 (7): 1073-82. (PMID: 20140443).

5. Williams-Gray CH, Foltynie T, Brayne CE, et al. Evolution of cognitive dysfunction in an incident Parkinson's disease cohort. Brain 2007; 130 (7): 1787-98. (PMID: 17535834).

6. Foltynie T, Brayne CE, Robbins TW, Barker RA. The cognitive ability of an incident cohort of Parking's patients in the UK. The Cam-PaIGN study. Brain. 2004; 127 (3): 550-60. (PMID: 14691062).

7. Meireles J, Massano J. Cognitive Impairment and Dementia in Parkinson's Disease: Clinical Features, Diagnosis, and Management. Frontiers in Neurology 2012; 3 (88): 1-15. (PMID: 22654785).

8. Grant WB. Epidemiology of disease risks in relation to vitamin D insufficiency. Prog Biophys Mol Biol 2006; 92 (1): 65-79. (PMID: 16546242).

9. Eyles DW, Burne THJ, John J McGrath JJ. Vitamin D, effects on brain development, adult brain function and the links between low levels of vitamin $D$ and neuropsychiatric disease. Front Neuroendocrinol 2013; 34 (1): 47-64. (PMID: 22796576)

10. Wetmore C, Cao Y, Pettersson RF, et al. Brain-derived neurotrophic factor: subcellular compartmentalization and interneuronal transfer as visualised with anti-peptide antibodies. Proc. Nall. Acad. Sci 1991; 88 (21): 9843-47. (PMID: 1946410).

11. Phillips HS, Hains JM, Armanini $M$, et al. BDNF mRNA is decreased in the hippocampus of individuals with Alzheimer's disease. Neuron 1991; 7 (5): 695 702. (PMID: 1742020).

12. Holsinger RM, Schnarr J, Henry P, et al. Quantitation of BDNF mRNA in human parietal cortex by competi- tive reverse transcription-polymerase chain reaction: decreased levels in Alzheimer's disease. Brain Res Mol Brain Res 2000; 76 (2): 347-54. (PMID: 10762711).

13. Howells DW, Porritt M, Wong JY, et al. Reduced BDNF mRNA expression in the Parkinson's disease substantia nigra. Exp Neurol 2000; 166 (1): 127-35. (PMID: 11031089).

14. Hossein-Nezhad A, Holick MF. Vitamin D for Health: A Global Perspective. Mayo Clin Proc 2013; 88 (7): 720-55. (PMID: 23790560).

15. Ahn JD, Kang H. Physical Fitness and Serum Vitamin D and Cognition in Elderly Koreans. J Sports Sci Med 2015; 14 (4): 740-6. (PMID: 26664270).

16. Llewellyn DJ, Lang IA, Langa KM, et al. Vitamin D and Risk of Cognitive Decline in Elderly Persons. Arch Intern Med 2010; 170 (13): 1135-41. (PMID: 20625021).

17. Peterson AL, Murchison C, Zabetian C, et al. Memory, Mood, and Vitamin D in Persons with Parkinson's Disease. J Parkinsons Dis 2013; 3 (4): 547-55. (PMID: 24081441).

18. Knekt $P$, Kilkkinen $A$, Rissanen $H$, et al. Serum vitamin $\mathrm{D}$ and the risk of Parkinson's disease. Arch Neurol 2010; 67 (7): 808-11. (PMID: 20625085).

19. Evatt $M L$, De Long MR, Khazai $N$, et al. Prevalance of Vitamin D Insufficiency in Patients With Parkinson Disease and Alzheimer Disease. Arch Neurol 2008; 65 (10): 1348-52. (PMID: 18852350).

20. Suzuki M, Yoshioka M, Hashimoto M, et al. Randomized, double-blind, placebo-controlled trial of vitamin D supplementation in Parkinson disease. Am J Clin Nutr. 2013; 97 (5): 1004-13. (PMID: 23485413).

21. Zhou Z, Zhou R, Zhang Z, et al. The Association Between Vitamin D Status, Vitamin D Supplementation, Sunlight Exposure, and Parkinson's Disease: A Systematic Review and Meta-Analysis. Med Sci Monit 2019; 25: 666-74. (PMID: 30672512).

22. Laske C, Stransky E, Leyhe T, et al. BDNF serum and CSF concentrations in Alzheimer's disease, normal pressure hydrocephalus and healthy controls. J Psychiatr Res 2007; 41 (5): 387-94. (PMID: 16554070).

23. Scalzo P, Kümmer A, Bretas TL, et al. Serum levels of brain-derived neurotrophic factor correlate with motor impairment in Parkinson's disease. J Neurol 2010; 257 (4): 540-5. (PMID: 19847468).

24. Faria MC, Goncalves GS, Rocha NP, et al. Increased plasma levels of BDNF and inflammatory markers in Alzheimer's disease. J Psychiatr Res. 2014; 53: 16672. (PMID: 24576746). 